\title{
Qualitative Analyse von Online Communities für Neuproduktentscheidungen
}

\author{
Rudolf R. Sinkovics $\cdot$ Elfriede Penz $\cdot$ Francisco Jose Molina Castillo
}

Eingegangen: 3. März 2008 / Angenommen: 15. November 2008 / Online veröffentlicht: 26. März 2009 (C) Springer-Verlag 2009

Zusammenfassung In zunehmendem Maße verwenden KonsumentInnen computer-basierte Kommunikationsformen, Bulletin-boards, Newsgroups, Chatrooms, Emailverteiler und Webseiten und sonstige online Formate um sich auszutauschen beziehungsweise um nach Produkten zu recherchieren und diese zu vergleichen. Online Communities haben sich als ausgezeichnete Möglichkeit erwiesen um allgemein verfügbares Wissen und Produkt Know-how KonsumentInnen und auch Unternehmen verfügbar $\mathrm{zu}$ machen. In der Praxis der Marketingforschung wird dieser Wissenspool jedoch noch unzureichend genutzt und Produktneugestaltung und Produktvariationsentscheidungen auf der Basis von Online Communities sind noch eine Seltenheit. Dieses Manuskript stellt Online Communities als eine kostengünstige und vielfältige Alternative für qualitative Marktforschung vor. Es werden Wege und Möglichkeiten diskutiert wie qualitative Information aus Online Communi-

R. R. Sinkovics ( $)$

Manchester Business School,

Booth Street West, M15 6PB Manchester, UK

E-Mail: Rudolf.Sinkovics@manchester.ac.uk

Tel.: +44-161-306 8980

URL: http://www.personal.mbs.ac.uk/rsinkovics/

E. Penz

Wirtschaftsuniversität Wien,

Augasse 2-6, 1090 Wien, Österreich

E-Mail: Elfriede.Penz@wu-wien.ac.at

Tel.: +43-1-31336 5102

URL: http://www.wu-wien.ac.at/imm/penz/

F. J. Molina Castillo

University of Murcia,

Campus de Espinardo, 30100 Murcia, Spain

E-Mail: fjmolina@um.es

Tel.: +34-968-367826

URL: http://www.marketing.um.es/professor/fjmolina ties für Analysen verfügbar gemacht werden können und wie diese als Grundlage für Neuprodukt- und Produktvariationsentscheidungen Verwendung finden können.

Schlüsselwörter Communities · Neuproduktentscheidungen · Qualitative Forschung

\begin{abstract}
Consumers are attending to computer-mediated communications, newsgroups, chat rooms, email list servers, personal World Wide Web pages, and other online formats to share ideas and obtain information about products. Online communities and virtual communities have emerged as new tools which represent a fascinating pool of collective experience and product know-how. However, the utilization and analysis of this body of knowledge for new product development and innovation is still at its infancy. This paper suggests that online communities offer a costeffective and rich platform for qualitative market-research. We explain how to identify and access online communities, and how to convert qualitative information available in online community sites into strategic input for new product decisions.
\end{abstract}

Keywords Communities - New product decisions · Qualitative research

\section{Einleitung und Motivation}

Das Internet hat sich als spannendes Medium für die Demokratisierung und Stimulierung der Kommunikation und des Wissenstransfers herausgestellt (vgl. Penz 2007). Im Gegensatz zu dem traditionellen ,one-to-many“ Marketing- 
Kommunikationsmodell für Massenmedien (Hoffman u. Novak 1996), erfolgt Marketingkommunikation in der hypermedialen, computer-mediierten Umwelt dezentral und organisiert sich rund um das Medium und den Kommunikationsinhalt. In zunehmendem Maße greifen KonsumentInnen auf computer-mediierte Kommunikationsformen als Grundlage für ihre Kaufentscheidungen zurück (Kozinets 2002). Durch Entwicklungen im Bereich der Informationstechnologie verfügen KonsumentInnen nun über neue Möglichkeiten zur Interaktion mit anderen KonsumentInnen, beziehungsweise zum Informationsaustausch und in Bezug auf Unternehmen zu einfacheren Beziehungsmanagement. Vielfach werden hier die Begriffe „,computer-basiert“, ,online“ oder „virtuelle Communities“ verwendet. Communities und computer-basierter Informationsaustausch mit anderen KonsumentInnen wird gemeinhin als objektiver und wahrhaftiger verstanden (Casaló et al. 2008). KonsumentInnen bieten in diesem online Zusammenhang Feedback an, welches über traditionelle Marktforschungsmaßnahmen üblicherweise nicht erfassbar ist. Aus diesem Grund eröffnet das netzwerkbasierte Computerzeitalter für Manager und Firmen neue Möglichkeiten hinsichtlich von Marktforschung, bzw. der Identifikation von KonsumentInnenverhalten und Produktentscheidungen (Kozinets 2002). Das gesteigerte Interesse bezüglich Lernen, Wissenstransfer und Informationsaustausch in Online Communities, sowie Möglichkeiten der Organisation des Management und der Organisation dieses Wissens für betriebliche Zwecke ist daher sehr verständlich (Dholakia et al. 2004).

In diesem Artikel wird ein qualitativer Ansatz zur Analyse von Daten aus Online Communities verfolgt. Die Motivation dazu folgt dem gesteigerten Interesse gegenüber qualitativen Forschungsmethoden wie etwa durch Marschan-Piekkari u. Welch (2004) in einem Forschungsband zur Bedeutung qualitativer Methoden für internationale Geschäftstätigkeit bekundet. Sinkovics et al. (2005) argumentieren, dass qualitative Methoden gute Hilfestellung dabei leisten, Strukturen hinter quantitativen Daten zu erkennen oder eine Möglichkeit anbieten mit der Informationsflut quantitativer Daten konstruktiv umzugehen. Qualitative Methoden stellen nach ihrer Meinung ein Hilfsmittel für das Verständnis multidimensionaler globaler Phänomene dar und bieten im Regelfall mehr Flexibilität. Dies kommt etwa dadurch zum Ausdruck, dass qualitative Methoden sich gut den Forschungsproblemen anpassen lassen anstatt umgekehrt, d. h. dass das Forschungsproblem sich nach der Methode richten müsste (Sinkovics et al. 2005).

Zusätzlich zielt dieser Artikel darauf ab, das Phänomen „Online Communities“ zu erfassen. Es bietet sich ein qualitativer Ansatz an, weil die Forschungslandschaft in diesem Kontext noch weit offen ist und eine explorative Vorgangsweise bei der weiteren Konzeptionalisierung des Phänomens helfen kann (Ghauri u. Firth 2009). Zudem hilft ein formalisierter qualitativer Ansatz die Glaubwürdigkeit in die gewonnenen Untersuchungsergebnisse $\mathrm{zu}$ erhöhen (Sinkovics et al. 2008).

KonsumentInnen bringen sich immer öfter in Online Communities ein um Produkte zu evaluieren oder Produktentscheidungen informativ zu unterstützen und neue Ideen für Produkte zu entwickeln. In diesem Artikel wird eine qualitative, textanalytische Vorgehensweise eingesetzt. Kommentare von NutzerInnen eines online Spieleportals werden hinsichtlich ihrer Verwendbarkeit für Neuproduktentscheidungen beziehungsweise Produktvariationsentscheidungen untersucht. Konkret untersuchen wir welche Informationen von Online Communities im Bereich „online Spiele - MMORPGs (massively multiplayer online role playing games $=$ Computerrollenspiel mit gleichzeitiger, virtueller Interaktion der SpielerInnen)“ erhoben werden können und welche Vorteile die Spieleherausgeber und -produzenten von Informationen haben können, die in Online Communities zur Verfügung gestellt werden.

Der weitere Aufbau dieses Artikels ist wie folgt. Zunächst werden die konzeptionellen Grundlagen von Online Communities vorgestellt, dann wird die Theorie des sozialen Austauschs behandelt. Die Theorie kann helfen, die Motivationen von Community-Mitgliedern zur Teilnahme in Online Communities und ihren Beitrag zur Produktentwicklung von MMORPGs zu erklären. Anschließend gehen wir der konzeptionellen Frage nach wie unterschiedliche Community-Formen und Nutzer-Interaktionen für Marketingzwecke verwendet werden können. Dabei fokussieren wir auf die Neuproduktentwicklung beziehungsweise auf Produktvariationen. Im konzeptionellen Teil unterscheiden wir zwischen verschiedenen Community-Formen, konkret zwischen virtuellen Communities und virtuellen Welten und stellen anschließend verschiedene Online Communities in Bezug auf Nutzen und Funktion graphisch dar. In den folgenden Abschnitten stellen wir ein empirisches Beispiel vor, welches veranschaulicht, wie qualitative, textbasierte Informationen, die aus Online Communities gewonnen werden können unter Computereinsatz (CAQDAS) analysiert werden können, und wie diese Informationen in Marketingentscheidungsprozesse einfließen können. Der Artikel endet mit einer Darstellung von Forschungsbeschränkungen der gegenwärtigen Studie und bietet Perspektiven für zukünftige Forschungsarbeiten an.

\section{Konzeptioneller Rahmen}

\section{Darstellung von Online Communities}

Online Communities sind laut Definition von Rheingold (1993) "social aggregations that emerge from the Net when enough people carry on those public discussions 
long enough, with sufficient human feeling, to form webs of personal relationships in cyberspace". Literatur zum Thema Online Communities entwickelt sich in letzter Zeit stärker, insbesondere im Bereich der Konsumentenverhaltensforschung, doch die Marketing-, und Informationssystem-Perspektiven haben noch keine umfassend gültige Definition zu Online Communities hervorgebracht. Muniz u. O'Guinn (2001) argumentieren, dass Online Communities Kontexte darstellen, innerhalb derer KonsumentInnen diskutieren. Diese Diskussionen können konvergierende Ziele bezüglich der Informationsweitergabe und Produkt- oder Markenbeeinflussung durch andere KonsumentInnen haben. Kollock u. Smith (1999) sehen Online Communities als soziale Netzwerke, deren Mitglieder durch Computerunterstützung miteinander in Verbindung treten. Andere AutorInnen streichen besonders die Beziehungsdimension der Mitglieder hervor. Preece (2000) argumentiert, dass Online Communities aus Menschen gebildet werden, deren soziale Interaktion der Befriedigung ihrer eigenen Informations- und Kommunikationsbedürfnissen gilt und wo Computersysteme die soziale Interaktion steuern, sowohl über formale als auch über informale Regeln. Andrews et al. (2002) stellen fest, dass es sich bei Communities nicht um physische Orte handelt, sondern um ein Geflecht sozialer Beziehungen. Obwohl bestimmte Mitglieder einer demographischen Gruppe ähnliche Interessen, Bedürfnisse und Ziele teilen können, bedingt dies nicht notwendigerweise eine Community. Nur wenn diese Gruppe ein Netzwerk sozialer Austauschbeziehungen bildet, ist damit eine Community konstituiert. Diese sozialen Interaktionen sind vorwiegend online und passieren in virtuellen Kontexten durch die Übermittlung von Inhalten durch Gruppenmitgliedern (Jang et al. 2008). Das Mitteilen von Gruppenzielen und der Ideenaustausch wird dabei weder geographisch noch durch ethnische Aspekte eingeschränkt (Hsu u. Lu 2007).

Kozinets (1999) nähert sich dem Thema der Online Communities von einer Marketing und Managementperspektive an. Online Communities sind dabei jene Zusammenschlüsse von Menschen, deren nicht-physische Austauschbeziehungen auf gemeinsamem Enthusiasmus oder Wissen für bestimmte Konsumaspekte oder Produkte beruhen. Online Communities unterscheiden sich von direkten und physischen Begegnungen durch den Mangel an expliziten physischen Schlüsselinformationen. Community-Mitglieder haben die Möglichkeit ihre Identität zu verändern, sowie soziale Ordnung und Zweck zu definieren. Brown et al. (2007) zeigen, dass der Informationsaustausch von Mitgliedern in einem online Netzwerk anders als in der realen Welt verläuft und weisen darauf hin, dass verschiedene Internet-Technologien wie Usenet-Gruppen, Listservers, schwarze Bretter und Webseiten für Online Communities Verwendung finden.
Armstrong u. Hagel (1996) klassifizieren Online Communities in unterschiedliche Kategorien, basierend auf Transaktionen, Interesse, Phantasie und Beziehungstypen. Winkler u. Mandl (2004) unterscheiden zwischen asynchroner und synchroner computer-mediierter Kommunikation. Dabei beinhalten asynchrone Formen Webforen, Newsgruppen und Mailinglisten. Synchrone computerbasierte Kommunikation beinhaltet Chatrooms und „multi user dungeons" (MUDs). ${ }^{1}$ Der empirische Teil dieses Artikels beschäftigt sich mit Forumsdiskussionen (asynchrone computer-mediierte Kommunikation) zu einem MMORPG, konkret World of Warcraft (WoW). WoW stellt eine synchrone computer-mediierte Form einer Virtual Community dar, weil deren Nutzer die Möglichkeit haben miteinander zur selben Zeit in Kontakt zu treten. Dies geschieht durch gemeinsames Spielen (zum Beispiel ,raids"), oder durch Kommunikation im Spiel (zum Beispiel durch „whispers“ als private, real-time Interaktionsmöglichkeiten, oder durch „teamspeak“, einer Sprachkonferenzsoftware).

Eine weitere Möglichkeit Online Communities darzustellen bieten wir in Abb. 1. Darin werden Beispiele für Online Communities entlang zweier Dimensionen dargestellt, einerseits nach geschäftlichem und sozialem Nutzen (vertikale Dimension) und andererseits nach kreativem/spielerischem und funktionalem Austausch (horizontale Dimension).

Wie der Abbildung zu entnehmen ist, sind beispielsweise eine Reihe von Open-Source Projekten im Quadrant angesiedelt der durch kreativen/spielerischen Austausch und geschäftlichen Nutzen aufgespannt wird. Online Communities wie Sourceforge oder Apache funktionieren unter Beteiligung von vielen Programmierern, deren individuelle Nutzen an der Community teilzunehmen mit der Zahl der Mitglieder anwächst. Bei Myspace, Facebook und Flickr andererseits, steht mehr der funktionale Austausch (Fotos oder Adressdaten), in Kombination mit dem sozialen Nutzen der Vernetzung im Vordergrund.

\section{Sozialer Austausch und Neuproduktentscheidungen}

Die Theorie des sozialen Austauschs erklärt das Verhalten von Gruppenmitgliedern und auch zwischen mehreren Gruppen. Es erklärt, warum Menschen einander unterstützen und auch warum es Informationsaustausch zwischen Gruppenmitgliedern gibt (Cropanzano u. Mitchell 2005). Die Theorie des sozialen Austauschs impliziert für den virtuellen/digitalen Kontext dass die Interaktion sowohl für den Informationsproduzenten als auch den Empfänger lohnenswert sein muss (Anderson et al. 1999; Emerson 1976). Belohnung ist, wie Wasko u. Faraj (2005) betonen,

\footnotetext{
${ }^{1}$ Rollenspiel das auf einem zentralen Computer (Server), auf dem sich mehrere Spieler (Mudder oder MudHead) gleichzeitig einloggen können (http://de.wikipedia.org/wiki/Multi_User_Dungeon).
} 
Abb. 1 Graphische Repräsentation des funktionalen und geschäftlichen Nutzens ausgewählter Online Communities

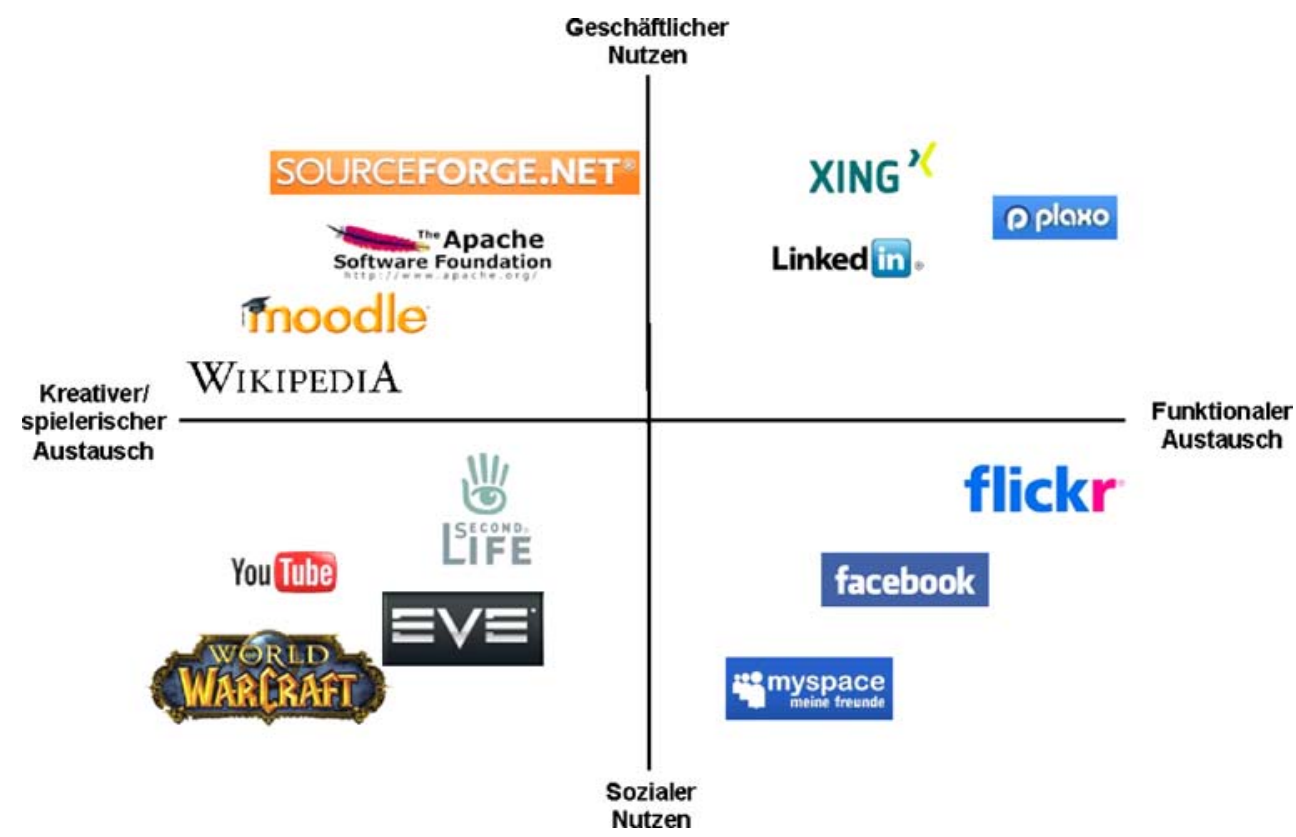

wichtig, weil KonsumentInnen in Online Communities Zeit und Mühen investieren, die nur mittelbaren und nicht unmittelbaren Nutzen für die Beitragsleistenden stiften. Obwohl es in jüngster Zeit ein zunehmendes Interesse und eine zunehmende Zahl von Forschungsarbeiten zu sozialem Austausch (Hemetsberger 2002) und offenen Informationsaustausch und Innovation in Online Communities gegeben hat (von Hippel u. von Krogh 2003), existieren nach wie vor eine Reihe von Fragen bezüglich des Nutzens von Community-Beteiligung und der Attraktion der Community-Besucher (Gu et al. 2007).

Die Theorie des sozialen Austauschs bietet eine Vielzahl theoretischer Ansatzpunkte für die Analyse von innovativem Nutzerverhalten innerhalb des Online-CommunityKontextes an. Andrews et al. (2002) bieten einen konzeptionellen Erklärungsrahmen für demographische Gruppen an, die sich gegenüber Online-Community-Interaktion resistent zeigen. Sie weisen darauf hin, dass gute Sozialisationsfaktoren und Community-Klima ausgesprochen wichtig für das Vertrauen zwischen Mitgliedern und für ihre Erwartungen hinsichtlich des Teilens von Information sind.

Kozinets (2002) erläutert, dass die Teilnahme in Online Communities KonsumentInnen hilft, sich über Produkte, Marken und Firmen zu informieren. Durch den Austausch und die Kooperation mit ähnlich gesinnten Personen, Freunden, KollegInnen, wird produktspezifisches Wissen und Produktunterstützung zur Verfügung gestellt. Dieses Wissen kann Ideen generieren und in den Neuproduktentwicklungsprozess übergeführt werden (Franke u. Piller 2003; Füller et al. 2007). Füller et al. (2006) entwickeln ein community-basiertes Innovationsmodell für Online Communities und schlagen vor, wie diese gestaltet werden müssen und welche Funktionalitäten diese bieten sollten, um Mitgliederinteraktion $\mathrm{zu}$ stimulieren und wertvollen Input für Neuproduktentwicklungen generieren zu können. Online Communities helfen in der Bedarfsidentifikation verschiedener Gruppen (Casaló et al. 2008) und helfen in der raschen Verbreitung von Produktwissen und Produktwahrnehmungen, was gerade für neu auf den Markt gebrachte Produkte von Bedeutung ist (Dholakia et al. 2004).

Trotz dieser attraktiven Eigenschaften von Communities wurden sie bislang nur selten in die Überlegungen zur Wertschöpfung in der Neuproduktentwicklung eingesetzt. Bislang beschränkte sich die Nutzung von Online Communities auf die klassischen Aspekte der Marktforschung (Füller et al. 2006). Balasubramanian u. Mahajan (2001) diskutieren die Rolle und die Marketingimplikationen von Online Communities. Füller et al. (2006) schlagen vor, dass Online Communities für KonsumentInnen durch die Einbeziehung eines sogenannten ,toolkit approaches“ auch die Möglichkeit des Designs und der Entwicklung eigener Produkte anbieten könnten (Franke u. Piller 2004, 2003; von Hippel 2001). Wie in Abb. 2 ersichtlich, können unterschiedliche Formen von Communities in unterschiedlichen Phasen der Neuproduktentwicklung eingesetzt werden. Die Formen werden im Detail im nächsten Abschnitt besprochen.

\section{Weitere Formen von Communities}

Neben Online Communities wurden in der Literatur auch sogenannte ,virtuelle Communities“ und „,virtuelle Welten“ eingeführt. Dabei handelt es sich um spezielle Formen von Communities. Im folgenden werden sie kurz beschrieben. 


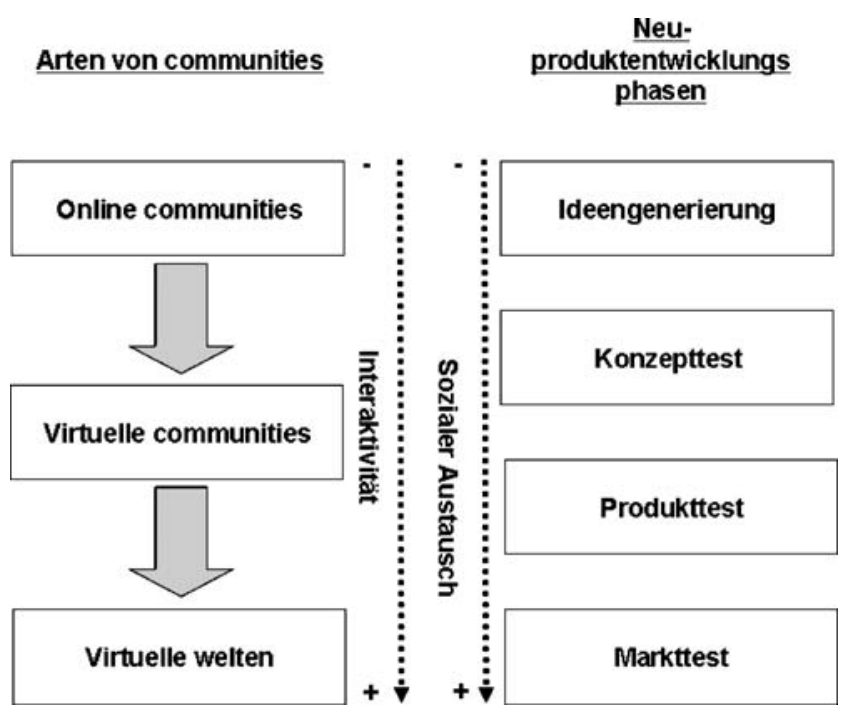

Abb. 2 Konzeptionelle Darstellung der Korrespondenz der Community Formen und den Phasen im Produktentwicklungsprozess

\section{Virtuelle Communities}

Bei einer virtuellen Community handelt es sich um einen Zusammenschluss von Menschen, die ein gemeinsames Interesse verfolgen und aus diesem Grund regelmäßig und in organisierter Form über das Internet interagieren (Ridings et al. 2002). Mitglieder von virtuellen Communities tauschen Information und Wissen aus und sind in soziale Interaktionsprozesse involviert (Chiu et al. 2006). Bagozzi u. Dholakia (2002) definieren virtuelle Communities als soziale Räume in digitalen Umgebungswelten, die Gruppenbildungen und deren Erhalt primär durch kontinuierliche Kommunikationsprozesse erlauben. Virtuelle Communities unterscheiden sich von traditionellen Communities dadurch, dass Beteiligung und Kommunikation in virtuellen Communities oft auf freiwilliger Basis erfolgt, während im anderen Fall Mitgliedschaft auf Basis von deskriptiven Faktoren wie (Geburtsort, Wohnort, etc.) vorgeschrieben werden kann.

Virtuelle Communities sind im Gegensatz zu vielen anderen online Gruppen auch selbsterhaltend. Mitglieder treten in dynamische soziale Interaktionen ein (Schuler 1996), und werden daher vielfach als Manifestationen der Anpassungsschwierigkeiten, die man in der realen Welt vorfindet, bezeichnet (Robins 1996). Die sozialen Interaktionen wurden bislang unter soziologischen, psychologischen und anthropologischen Gesichtspunkten analysiert. Nach Blanchard (2008), ist das „Gefühl der virtuellen Zusammengehörigkeit“" ein Hauptdifferenzierungskriterium das virtuelle Communities von anderen Ausprägungen unterscheidet. Koh u. Kim (2003) streichen dabei drei Dimensionen hervor: (1) Mitgliedschaft - Personen erleben ein Gefühl der Zugehörigkeit zu ihrer virtuellen
Community, (2) Einflussnahme - Community-Mitglieder beeinflussen andere Community-Mitglieder, und (3) Tiefe - Community-Mitglieder erleben ein „Flow“ Gefühl während ihrer Anwesenheit in der virtuellen Community, d. h. sie tauchen gewissermaßen tief in die virtuelle Umgebung ein.

Virtuelle Communities können entweder ein enges oder loses Netzwerk repräsentieren, sie können zu sozialen oder auch kommerziellen Gründen geschaffen werden. Hagel u. Armstrong (1997) weisen darauf hin, dass virtuelle Communities vier spezifische Arten von KonsumentInnenBedürfnissen erfüllen: Ressourcenteilung, Etablierung von Beziehungen, Handel und kommerzielle Transaktionen sowie das Teilen und Erleben von Fantasien. Die Kombination von verschiedenen Faktoren in Online Communities, wie etwa der zwischenmenschlichen Komponente, der Kommunikation zwischen Gruppenmitgliedern, kann bei geeigneten online Firmenstrategien und direkter Werbung auch eine Grundlage für Nachfragesteigerung sein (Miller et al. 2009).

\section{Virtuelle Welten}

Die informations- und kommunikationstechnologischen Entwicklungen der letzten Jahre haben es ermöglicht, dass sich Menschen in virtuellen Welten zusammenfinden, dort ökonomische und soziale Realitäten entwickeln, mit anderen kommunizieren und in komplexe soziale und virtuelle Aufgabenbereiche eindringen. Diese Interaktionen können mittlerweile in dreidimensionalen Umgebungen ablaufen (England u. Gray 1998) und sind durch Sprachund Textkommunikation äußerst realitätsnah. Teilnehmer an virtuellen Welten können ihre eigene Identität entwickeln (Avatare) und diese zur Interaktion mit Dritten verwenden.

Es gibt im Internet eine Reihe von virtuellen Welten. Die meisten fallen in die Kategorie der ,Massively Multiplayer Online" (MMO) games. MMORPGs im speziellen, wie etwa Second Life oder World of Warcraft sind nicht bloß Spiele in virtuellen Welten, man kann diese vielmehr als komplexe Online Communities sehen, welche weitere online Communities hervorbringen (zum Beispiel Forumsdiskussionen), welche für Forscher exzellente Laborbedingungen für soziale Forschungsfragen bieten (Mennecke et al. 2007).

Qualitative Analysemethoden für Online Communities

Hinsichtlich der Marktforschungsmethoden gibt es für die Untersuchung von Online Communities verschiedene Möglichkeiten, die teilweise parallel zu den Methoden der Marktforschung im Internet gesehen werden können. 


\section{Online-Delphi}

Die Methode Online-Delphi versucht verlässliche Informationen zu Sachverhalten zu generieren, in denen ein ungesichertes oder unvollständiges Wissen vorliegt. Die Methode wird auch benutzt um Aussagen über zukünftige Ereignisse oder Entwicklungen zu treffen, oder in der Marktwirtschaft um die Marktentwicklung einer neuen Erfindung innerhalb einer Branche abzuschätzen (Okoli u. Pawlowski 2004). Befragungen laufen über einen Zeitraum bis zu einigen Monaten in denen die Befragten wenigstens zweimal befragt werden. Die Methode ähnelt der Panel-Umfrage, findet nur nicht mehrmals zum gleichen Thema statt und ist in der Lebensdauer stark eingeschränkt (Rowe u. Wright 1999).

\section{Netnography}

Die Netnography basiert auf den Grundzügen der Ethnographie, also der Beobachtung des Verhaltens von Gruppen und ihrer einzelnen Mitglieder durch die direkte Beteiligung des Forschers (Hitzler 2007). Nicht die einzelnen Personen selbst sind der Untersuchungsgegenstand, sondern die im Internet beobachtbare Konversation und soziale Interaktion. Mit dieser Methode lässt sich herausfinden, wie die Mitglieder einzelner Online Communities über bestimmte Themen denken, wie sie einzelne Produkte und Marken bewerten bzw. welche Themen in der Community den Schwerpunkt bilden (Beckmann u. Langer 2007). Die von Kozinets (1999) vorgestellte Netnography Methode baut auf einem vierstufigen Prozess auf. (1) Zugang zu der Community (Cultural Entrée), (2) Sammlung und Analyse der Daten, (3) Interpretation der Daten, (4) Beachtung ethischer Richtlinien der Forschung. Sofern die mittels Netnography gewonnen Erkenntnisse verallgemeinert werden sollen, empfiehlt es sich, die Ergebnisse mittels anderer Marktforschungsmethoden, wie zum Beispiel mittels Fokusgruppen oder persönlichen Interviews, zu „triangulieren“ (Ghauri u. Grønhaug 2005).

\section{Analyse von online generiertem Text}

Für die Analyse von textbasierten Untersuchungsdaten, wie sie etwa aus qualitativen Experteninterviews erstellt werden (Aghamanoukjan et al. 2007), liegen eine Reihe von konzeptionellen Beiträgen und methodischen Befunden vor (vgl. di Gregorio 2007; Kuckartz 2007; Sinkovics u. Penz 2007; Sinkovics et al. 2008). Penz u. Sinkovics (2005) schlagen Forschungsstandards vor, deren Anwendung im Forschungsprozess und insbesondere im Zusammenhang mit der Analyse qualitativer Textdaten mithelfen soll, Kritikpunkten der mangelnden Übertragbarkeit, Verlässlichkeit und Glaubwürdigkeit, sowie Bestätigungsfähigkeit qualitativer Textdaten entgegenzuwirken (Guba u. Lincoln 1989).
Durch die Berücksichtigung dieser Aspekte im qualitativen Forschungsprozess wird die Logik des Forschers transparent gemacht. Zudem erhöht der Einsatz computergestützter Methoden die Vertrauenswürdigkeit der qualitativen Analyse (Sinkovics et al. 2005; Steinke 2007). Eine CAQDASbasierte Textanalyse stellt eine besonders geeignete Analysemethodik dar, weil durch Online Communities eine Vielzahl kodifizierter Textdaten vorliegen. Sie verbessert Forschungseffizienz, als auch intersubjektive Nachprüfbarkeit und Verallgemeinerbarkeit der Erkenntnisse (Steinke 2007).

\section{Methodologie}

Im Folgenden wird die Methodologie besprochen. Zunächst wird die Untersuchungseinheit in Bezug auf die Forschungsfragen definiert. Danach folgen die Darstellung des Untersuchungsmaterials sowie die Durchführung der Analyse. Zur Analyse des textbasierten Materials aus Webforen wurde die Netnography Methode herangezogen, die sich gut mit der „Grounded Theory“ verbinden lässt, da beide Ansätze eine ständige Interaktion des Forschers mit dem Analysematerial ermöglichen. Abschließend werden exemplarisch einige Ergebnisse der Analyse diskutiert.

Forschungsfragen und Untersuchungseinheit

Wir greifen auf Texte aus Webforen zurück und untersuchen diese inhaltsanalytisch, unter Berücksichtigung von zwei konkreten Forschungsfragen. Einerseits soll herausgefunden werden, welche Informationen Webforen zum Thema Onlinespiele (MMORPGs) generell liefern können. Andererseits wird diese Frage dahingehend erweitert dass wir versuchen herauszufinden wie Unternehmen und Teilnehmer in Webforen von diesen Informationen profitieren können.

Die Untersuchungseinheiten in der Analyse sind daher Webforen mit Kommunikationsaktivitäten und Informationsaustausch von Online Spielern und nicht die Teilnehmer und Diskutanten auf diesen Webforen selbst. Die Webforen bieten in den verschiedenen Threads nicht nur Diskussionen zu spielspezifischen Themen an; oft gibt es auch Bereiche, in denen über nicht spielbezogene Themen aller Art diskutiert wird.

\section{Material}

Die Auswahl der Webforen erfolgte hauptsächlich nach der Popularität der Spiele. Für den deutschsprachigen Raum sind das die Spiele World of Warcraft, Guild Wars, Der Herr der Ringe Online und EVE Online. Zusätzliche Auswahlkriterien waren die behandelten Themen, die Anzahl der Posts, die Verfügbarkeit und der Zugang zu 
den Spielen. Auf Grund dieser Kriterien wurden die Spiele World of Warcraft (entwickelt von Blizzard Entertainment) und EVE Online (CCP Games, Island) zur näheren Analyse ausgewählt.

World of Warcraft zählt zu den weltweit meistverkauften Online Rollenspielen. Spieler wählen zwischen zwei großen Fraktionen, der Horde und der Allianz, mit jeweils mehreren verfügbaren Rassen und Charakterklassen. $\mathrm{Zu}$ World of Warcraft werden auch laufend Erweiterungen angeboten, wie zuletzt „Wrath of the Lich King“ im November 2008 (http://eu.blizzard.com/de/press/081120.html), welche 2,8 Millionen Exemplare innerhalb der ersten 24 Stunden verkaufte.

Bei EVE Online geht es hauptsächlich um Kämpfe und Handel im Weltraum. Bei diesem Spiel sind üblicherweise viele Spieler gleichzeitig beteiligt, so wurde im Dezember 2007 mit 41.688 gleichzeitigen Onlinespielern ein neuer Rekord aufgestellt. EVE Online unterscheidet sich außerdem von anderen bekannten MMORPGs, durch seine ausserordentliche Komplexität.

Nicht immer ist es möglich, Details zu und Diskussionen in Webforen zu erheben, da Forumbetreiber oft keine Einsicht in genaue Benutzerzahlen, verfasste Themen oder Beiträge geben. Einen weiteren Problempunkt stellen Abweichungen und Verfehlungen von Themenstellungen dar. Immer wieder kommt es vor, dass der Inhalt von Themen nicht zur Überschrift passt und vice versa. Auch verfügen manche Foren über keine Suchfunktion, was das Aufspüren relevanter Threads und Beiträge bei der beträchtlichen Fülle an Informationen und Daten erschwert. Tabelle $1 \mathrm{gibt}$ abschließend einen Überblick über die Beiträge, die Themen, und die Benutzer der Webforen „World of Warcraft“ und „EVE Online“.

Durchführung

Innerhalb der Webforen wurde im Dezember 2007 von zwei Forschungsassistenten nach relevanten Themenberei- chen gesucht. Die Texte, verfügbar in Form von Beiträgen (sogenannten ,postings"), wurden anschließend für die softwaregestützte Analyse aufbereitet. Die Auswahl der Themenbereiche sowie die Auswahl der Beiträge wurden im Team besprochen und festgelegt. Die für die Analyse ausgewählten Themenbereiche (sogenannte threads) sind „Motivation für Onlinespiele“ und „Kritik am Spiel“, da diese Themenbereiche für Spielehersteller (zum Beispiel Entwickler) relevant sein können, um Spiele weiter zu entwickeln beziehungsweise anzupassen. Die Beiträge sowie alle daran anschließenden Antworten wurden mittels des Softwareprogramms NVivo (Richards 2000) nach der Netnography Methode analysiert (siehe Tabelle 2 und 3).

Beim Thema „Motivation“ geben die Mitglieder ihre Beweggründe an, weiterhin Onlinespiele zu spielen oder mit dem Spielen anzufangen. In den meisten Fällen beziehen sich die Beteiligten auf ein konkretes Onlinespiel beziehungsweise auf einen Spieletitel. Konkrete Diskussionen über Onlinespiele im Allgemeinen (ohne Beschränkung auf ein bestimmtes Spiel) sind eher die Ausnahme. Bei diesen Threads können Spielehersteller auf relativ einfache und schnelle Weise in Erfahrung bringen, wo ihre Stärken liegen beziehungsweise was bei den Spielern besonders gut ankommt.

Threads, die sich mit „Kritik am Spiel“ befassen, werden von Mitgliedern und interessierten Außenstehenden besonders gern gelesen, was sich auch bei der Anzahl an $\mathrm{Zu}$ griffen ausdrückt. Spieler bemängeln häufig das Spiel an sich (schlechte Grafik, viele Bugs, usw.), äußern Kritik auf Grund technischer Probleme (Serverüberlastung, schlechte Verbindung, hohe Systemanforderungen usw.) oder sind unzufrieden mit den Leistungen des Unternehmens (kein Support, langsame Wartung, usw.). Auffallend ist, dass kritische Beiträge in fast allen Threads, auch in denen, die sich mit anderen Themen beschäftigen, zu finden sind.

Die Auswahl und Analyse der Threads stellt einen wichtigen, aber schwierigen Schritt in der Analyse dar. Zahlreiche Webforen verfügen über keine (erweiterte)

Tabelle 1 Eigenschaften der Webforen „World of Warcraft“ und „EVE Online“

\begin{tabular}{|c|c|c|c|c|c|}
\hline & $\begin{array}{l}\text { Anzahl der } \\
\text { Beiträge }\end{array}$ & $\begin{array}{l}\text { Anzahl der } \\
\text { Themen }\end{array}$ & $\begin{array}{l}\text { Anzahl der } \\
\text { Benutzer }\end{array}$ & $\begin{array}{l}\text { Beiträge pro } \\
\text { Thema } \\
\text { (Mittelwert) }\end{array}$ & $\begin{array}{l}\text { Beiträge pro } \\
\text { Benutzer } \\
\text { (Mittelwert) }\end{array}$ \\
\hline \multicolumn{6}{|l|}{ World of Warcraft } \\
\hline Deutschsprachige Website wow.gamona.de & 1301096 & N/A & 105511 & N/A & 12,33 \\
\hline Deutsche World of Warcraft Website inWoW.de & 1559577 & 104192 & 73640 & 14,97 & 21,18 \\
\hline Deutsche Fansite WOW Source & 37770 & N/A & 6350 & N/A & 5,95 \\
\hline \multicolumn{6}{|l|}{ EVE Online } \\
\hline Fansite EVE Germany & 167446 & 10789 & 6981 & 15,52 & 23,99 \\
\hline Forum für EVE Online 4players.de & 16648 & N/A & 2974 & N/A & 5,60 \\
\hline Community Website EVE-World.de & 3463 & 464 & 1683 & 7,46 & 2,06 \\
\hline
\end{tabular}

Anmerkung: Information zum offiziellen Deutschen Webforum (WOW) nicht erhältlich 
Tabelle 2 Themen „Motivation um MMORPGs zu spielen“

\begin{tabular}{|c|c|c|c|}
\hline Webforen & Themen & Antworten & Hits \\
\hline \multicolumn{4}{|l|}{ World of Warcraft } \\
\hline Offizielles deutschsprachiges Forum & Motivation? & 20 & 1473 \\
\hline \multirow[t]{3}{*}{ Deutsche World of Warcraft Website inWoW.de } & Warum spielst du WoW? & 159 & 4616 \\
\hline & Was motiviert euch? & 62 & 2174 \\
\hline & Motivation? & 44 & 1862 \\
\hline \multicolumn{4}{|l|}{ EVE Online } \\
\hline Fansite EVE Germany & EVE macht Spaß, weil... & 44 & 1957 \\
\hline
\end{tabular}

Tabelle 3 Themen „Kritik am Spiel“

\begin{tabular}{|c|c|c|c|}
\hline Webforen & Themen & Antworten & Hits \\
\hline \multicolumn{4}{|l|}{ World of Warcraft } \\
\hline Offizielles deutschsprachiges Forum & Bessere Grafik bitte,... & 105 & 2159 \\
\hline \multirow[t]{2}{*}{ Deutschprachige Community-Seite wow.gamona.de } & Klassik Instanzen spielen? Fast unmöglich. . . & 36 & 2893 \\
\hline & Die Luft ist raus? & 49 & 3579 \\
\hline \multirow[t]{3}{*}{ Deutsche World of Warcraft Seite in WoW.de } & Nun hat es mich auch erwischt & 72 & 6453 \\
\hline & Return of the lags! & 48 & 1886 \\
\hline & Die dümmsten Antworten der $<\mathrm{GM}>$ 's & 28 & 2974 \\
\hline \multicolumn{4}{|l|}{$\begin{array}{l}\text { EVE Online } \\
\text { EVE Online }\end{array}$} \\
\hline EVE Online & & & \\
\hline \multirow[t]{2}{*}{ Fansite EVE Germany } & Was nervt euch an Eve am meisten? & 86 & 4436 \\
\hline & EvE ödet mich nur noch an... & 491 & 22789 \\
\hline
\end{tabular}

Suchfunktion, beziehungsweise liefert die Suche unzufriedenstellende Trefferergebnisse. Viele der angeführten Threads wurden erst nach ,,manueller“ Suche gefunden. Ein weiteres Problem, das bei der Suche aufgetreten ist, betrifft die Überschrift und den Inhalt von Threads. Immer wieder stößt man auf Threads, deren Inhalt sich nicht mit der Überschrift bzw. dem Thema deckt. Sehr wahrscheinlich ist aber auch, dass Threads mit relevanten Beiträgen übersehen werden, weil die Überschriften nicht zum eigentlichen Thema der Threads passen.

Ergebnisse

Mittels NVivo ist es möglich, Textstellen zu kodieren und durch die Erstellung von so genannten Nodes (Knotenpunkten) relevante Informationen zu markieren und zu katalogisieren. Durch die Erstellung von Unterthemen zu den Hauptkategorien ergibt sich eine Baumstruktur, Nodes werden im späteren Verlauf verknüpft und Beziehungen können aufgezeigt werden.

Die Texte aus den Webforen wurden nach abgeschlossener Formatierung in NVivo importiert. Zwei Forschungsassistenten kodierten die Texte manuell, zusätzlich wurden „Coding Queries“ durchgeführt, bei denen die Absätze, welche die relevanten Suchbegriffe enthielten, automatisch kodiert wurden. Eine manuelle Nachkorrektur war dennoch notwendig, um zu vermeiden, dass gewisse Begriffe in einem falschen Kontext kodiert würden. Nach erfolgter Kodierung wurde die Lösung im Team diskutiert und schließlich ein Nodesystem angenommen. In der Analyse der Diskussionen der Foren wurden gemäß der Themen ,Motivation“ und „Kritik“ zwei Nodesysteme entwickelt. Die Nodestruktur für „Motivation“ enthält vier Tree Nodes und neun Unternodes, die Nodestruktur für „Kritik“ enthält sieben Tree Nodes mit 14 Unternodes (siehe Tabelle 4).

Die Analyse erfolgte mit Hilfe der NVivo Funktion „matrix coding query“, welche zuvor definierte Nodes zum Beispiel mit Attributen (zum Beispiel Forentyp: offiziell, inoffiziell; Spiel: WoW, EVE Online) oder mit anderen Nodes verknüpft. Als Ergebnis erhält man eine Auflistung von Referenzen und Anzahl von Quellen, die eine derartige Verknüpfung aufweisen und schließlich einen Vergleich über die Untersuchungseinheit hinweg ermöglichen.

Die Themen Motivation und Kritik am Spiel werden in der nachfolgenden Analyse konsolidiert und auf ihren Beitrag zur Weiterentwicklung von Spielen betrachtet. Zum Thema Motivation brachte diese Analyse beispielsweise, dass der wichtigste Faktor „Spa $\beta$ “ ist, der zumeist nicht näher erklärt wird, manchmal aber mit bestimmten Spieltätigkeiten oder mit einem anderen Node „Gleichgesinnte treffen“ zusammenhängen kann. Das bezieht sich gleichermaßen auf die verschiedenen Foren bzw. Spiele, wie die folgenden Zitate zeigen:

„WoW macht mir Riesenspaß, darum spiele ich es, sowie die meisten hier" (Alexikus, Forum wow.de); „Ich habe gestern Abend eine Nachtelfen-Druidin angefangen und da 
Tabelle 4 Nodesystem „Motivation“ und „Kritik“

Node Subnode Beschreibung

Motivation: Abtauchen in eine andere Welt

Gleichgesinnte treffen:

- Freunde

- Gilde

Spielecontent:

- Spieleinhalt

- Viele, abwechslungsreiche Aufgaben

Spieltrieb befriedigen:

- Spaß

- Sucht

- Zeitvertreib

Kritik: $\quad$ Contentupdates:

- Einführung neuer Features

- Einführung neuer Spielinhalte

- Verbesserungen

Grafik:

- Hardwareanforderungen

- Zeitgemäße Grafik

Geschwindigkeit:

- Verbindungsprobleme

Motivationsprobleme:

- Fehlender Anschluss an andere Spieler

- Langeweile

- Ständiges Wiederholen von Vorgängen

Wartung:

- Einhaltung der vorgegebenen Wartungszeit

- Zeitraum der Wartung

PvE (Player versus Environment)

vs PvP (Player versus Player)

Support:

- Supportgeschwindigkeit

- Supportqualität
Bei sämtlichen untersuchten Onlinespielen wurden die Spieleumgebung beziehungsweise die virtuelle Welt als Grund zum Spielen genannt. Viele Spieler bereisen gerne neue Gegenden oder erkunden Fantasiewelten.

Hier geht es darum, dass Freunde aus dem täglichen Leben auch spielen, oder dass sich im Spiel selbst Freundschaften gebildet haben.

Das Spielen in einer Gilde wird als Motivationsfaktor gesehen.

Bei allen untersuchten Spielen werden von manchen Spielern der Spieleinhalt und die gebotenen Funktionen gelobt.

Manchen Spielern ist wichtig, dass das Spiel Abwechslung bringt und eine Vielzahl von Aufgaben zu lösen ist.

Bei allen untersuchten Spielen ist der Spielspaß der am häufigsten genannte Grund.

Spieler stellen fest, dass es auch Sucht sein kann, die einen zum Spielen von Online Games treibt.

Reiner Zeitvertreib kann Spieler dazu motivieren, ihre Zeit mit Onlinespielen zu verbringen.

Spieler kritisieren mangelnde Contentupdates, d. h. neue Features wie beispielsweise neue Quests oder Gegenden werden nicht ausreichend ins Spiel eingebracht.

Spieler kritisieren, dass das Spielen auf hohen Grafikeinstellungen bzw. eine erneute Grafikengine zu hohe Anforderungen an die Hardware stellen würde.

Spieler vertreten den Standpunkt, dass die gebotene Grafik im Spiel nicht mehr zeitgemäß ist und verbessert werden müsste.

Hier werden Lags, häufiges Trennen der Verbindung und hohe Zugriffszeiten als Kritikpunkte genannt.

Spieler beanstanden, dass der Anschluss an andere Spieler nicht gegeben ist. Oft können keine Mitspieler für Gruppenmissionen gefunden werden oder die Bitte um Hilfe stößt auf taube Ohren. Es herrscht teilweise hohes Misstrauen oder stark ausgeprägtes Konkurrenzdenken.

Langeweile ist einer der Hauptkritikpunkte überhaupt. Spieler bemängeln vor allem langweilige Missionen, haben sich schlichtweg satt gespielt oder haben bereits alles erreicht, was zu erreichen möglich gewesen ist.

Hier werden die sich andauernd wiederholenden, monotonen Abläufe im Spiel, wie beispielsweise das als „Grinding“ bezeichnete ständige Töten von Monstern, kritisiert.

Es wird kritisiert, dass die Wartungsarbeiten zu lange andauern oder sie bemängeln den ungünstigen Zeitraum von Wartungsarbeiten, sie würden sich einen anderen Zeitraum wünschen.

Hier wird beklagt, dass der Verlust durch das Getötet werden durch andere Spieler sehr groß ausfällt. Der Spielspaß wird auch durch andere Spieler verdorben, die sinnlos Player Killing betreiben.

Die Geschwindigkeit, mit der der Support auf Anfragen von Spielern reagiert, steht hier im Mittelpunkt der Kritik. Die Qualität des Supports und die Leistungen der Supportmitarbeiter werden beanstandet. auch so viel los war in Teldrassil und Dunkelküste, kam sofort die Lust wieder. Das Spielen macht doch gleich mehr Spaß, wenn um einen herum viel los ist und nicht alles tot erscheint, wie z. B. im Outland" (BlackSun84, Forum wow.de).
Fehlt der Spaß beim Spielen, tritt „Langeweile“ ein, die bei den Forumsdiskussionen eine der häufigsten Kritikpunkte am Spiel war: „Letztes Jahr Silvester ist mein Account ausgelaufen und es war gut so. Es war einfach langweilig." (Forum www.gamona.de). Auch bei EVEon- 
line gibt es Kritik am Spiel: „Die Missionen, nicht besonders spannend. sollten auch dahingehend überarbeitet werden, dass einige vom bonusgeld/-zeit an deren schwierigkeit und im bezug auf kopfgelder angepasst werden. Die meisten Lvl2 drohnen missionen sind nur ätzend." (BaronTollTroll, Forum eveonline.de). Dabei finden sich im Bereich „Kritik“ Vorschläge für Verbesserungen oder Weiterentwicklungen der Spiele, wie ein Beitrag zum Spiel EVEonline zeigt:

„Missionen sind für mich schnarchlangweilig (das spannendste ist noch, zu schauen, ob der client den Lag durch Missis mit Attack-on-Enter und 40 NPCs aushält) geworden, Produktion und das ganze BPC-Geraffel zu umständlich. Minern ${ }^{2}$ könnte wieder interessant werden, wenn der Markt von NPC-Grenzen befreit wird;-)“(Archer79, eveonline.de).

In der von uns kodierten Kategorie „Motivation“ finden sich eine Reihe von Wünschen von Spielern bereits formuliert:

„Mir würde es gefallen, wenn die Spieler auch mehr Möglichkeiten hätten, die Spielwelt zu gestalten, etwa durch Bemalung der Raumschiffe, Graffiti an Stationen, Posformen, farbige Munition... Ich denke, eine stärker durch Spieler gestaltete Atmosphäre würde eher dem Geist von Eve gerecht werden, als eine nur von CCP designte Umgebung. CCP sollte dafür Tools - vielleicht geknüpft an Skills - zur Verfügung stellen. (WoW kann hier nicht Vorbild sein.) Vielleicht wird „Künstler" tatsächlich zu einem Beruf in Eve. Das würde zumindest die Vielfalt an Berufen in diesem Universum steigern, und gerade die Vielfalt, liebe Mitpiloten, ist auch eins der Dinge, die mir an Eve besonders gefallen." (Komaito, eveonline.de).

Wie in dem folgenden Statement ausgedrückt, macht das Spielen Spaß, weil man gerne Gleichgesinnte trifft (,Dafür haben wir in unserer Corp viel Spaß, minere gemeinsam, baue Schiffe, spare auf BPO's, kann handeln, bauen, minern, kämpfen, beschützen, Märkte beobachten, afkchatten $^{3}$ )“ (mikcor, Forum eveonline.de). Es entstehen durch das gemeinsame Spielen beziehungsweise durch die Mitgliedschaft in Gruppen („Gilde“ in WoW) Freundschaften im wirklichen Leben: „Ich habe auch ne Gilde, in der ich mittlerweile einige RL Freunde gefunden habe..." Permiso, Forum www.gamona.de), oder umgekehrt: „Ich habe WoW angefangen, weil ich es bei Freunden gesehen habe. Meine Gildenmember wohnen fast alle in der Nähe." amokfrosch, Forum wow.de).

Mit Hilfe des Tree Nodes „Abtauchen in eine andere Welt" wird deutlich, dass die Gestaltung der Online Spiele für die Spieler sehr wichtig ist:

\footnotetext{
${ }^{2}$ „Minern“ bezieht sich auf eine Spielaktivität in EVEOnline, nämlich durch Bergbau Geld zu verdienen.

${ }^{3}$ Anmerkung: ,afk = away from keybord“; Benutzer sitzt gerade nicht am Rechner.
}

„Das Spiel entwickelt sich in verschiedene Richtungen, ist richtig, denn Blizzard will ja auch die eSport-Klientel beschäftigen. Und das gelingt. Dass dieser Zweig des Spieles nicht jedem zusagt, war abzusehen. Weiterhin finde ich die Flugmounts eine der tollsten Neuerungen im Spiel, endlich kann ich die Welt erkunden wie ich es möchte - und nicht, wie Blizzard es mir mit den Trampelpfaden vorgibt." (Alinde, Forum wow.de).

Durch die Gegenüberstellung der Beiträge aus Foren zu unterschiedlichen Spielen wird auch deutlich, dass Spieler sehr oft Vergleiche zwischen Spielen anstellen. Insbesondere EVE online wird im Vergleich zu anderen Spielen als überlegen beschrieben. Das betrifft einerseits die Schwierigkeit (,EVE ist vorsätzlich nicht einsteigerfreundlich, nicht einfach und ist nicht für die breite Masse konzipiert"), andererseits die vielen und abwechslungsreichen Aufgaben: „Eve hingegen lebt mit von den vielen Möglichkeiten, die die Spieler in der Spielwelt haben, in Politik, Handel und Produktion, neben dem bloßen Itemsammeln." (Komaito, Forum eveonline.de).

\section{Implikationen und Forschungsausblick}

Die steigende Beliebtheit von Onlinespielen und der wachsende Anteil von MMORPGs am Computerspielemarkt macht es für Hersteller von Video- und Computerspielen immer attraktiver, sich diesem Genre zuzuwenden. Auch Herausgeber von Onlinespielen, die sich bereits erfolgreich am Markt etabliert haben sind daran interessiert, ihre Kunden zu halten. Dafür ist es notwendig, kontinuierliche Verbesserungen bieten zu können und auf Feedback von Spielern einzugehen.

Die angewandte Methode ermöglicht eine qualitative Analyse der gestellten Forschungsfragen und gibt dadurch einen Einblick in die Themen Online Gaming und Virtual Communities. Für Herausgeber von Computerund Videospielen (und im Speziellen für die Herausgeber von Onlinespielen) bietet es sich an, die im Internet bereits vorhandenen Informationen auszuwählen und zu interpretieren. Rückschlüsse zu den verschiedensten Themenbereichen, wie etwa den in diesem Artikel behandelten Themen „Motivation“ und „Kritik am Spiel“ können durch Aufbereitung von Forumsbeiträgen gezogen werden. Durch die Fülle der Informationen erscheint im Besonderen eine softwaregestützte Analyse, wie etwa NVivo, geeignet. Die Methode der Netnography ist ein praktisches Analysewerkzeug, welches nahezu völlige Freiheit in der Gestaltung des Forschungsdesigns gewährleistet. Durch die Möglichkeit, Nodes immer weiter anzupassen und auszubauen, neue relevante Textstellen einzubinden und miteinander $\mathrm{zu}$ kombinieren, kann die qualitative Analyse jederzeit um zusätzliche Inhalte erweitert werden. Dadurch stellt sich 
aber auch das Problem, dass die Analyse keinen eigentlichen Endpunkt erreicht. Hier ist der Forscher gefordert, den Überblick nicht zu verlieren und den gesetzten Fokus einzuhalten.

Bezug nehmend auf die erste Forschungsfrage, welche Informationen Web Communities zum Thema Onlinespiele liefern, stellt sich heraus, dass diese eine Fülle von Informationen, welche die Spieler von Online Games untereinander im Internet austauschen, bieten. Die in den Webforen gesammelten Informationen und Daten reflektieren das Wissen aller Mitglieder, die sich innerhalb der Communities an Diskussionen beteiligen. In diesen virtuellen Communities wird Wissen kommuniziert, generiert, präsentiert und genutzt. Für die Hersteller sind sie insofern zweckdienlich, als dass diese Informationen jederzeit aktuell und abrufbereit sind.

Spieler motiviert bei Online Spielen, Spaß zu haben, mit Freunden gemeinsam zu spielen oder neue Freunde zu gewinnen, die virtuelle Umgebung zu erkunden, Gilden zu gründen, Spielfiguren zu gestalten, gegen andere Spieler zu kämpfen, virtuelle Items zu erstehen oder zu verkaufen und Missionen zu erfüllen. Das heißt die Spieler wünschen sich bei einem Onlinespiel Vielfalt und jeder will auf seine Kosten kommen.

Nimmt man als Beispiel das international sehr erfolgreiche World of Warcraft, so wird klar, dass von Blizzard tatsächlich versucht wird, für jeden etwas zu bieten. So gibt es im Spiel zum Beispiel mittlerweile zehn spielbare Rassen, anpassbare Hautfarben, Gesichter, Frisuren und Haarfarben, sowie anzeigbare Charaktertitel und Reputationspunkte, sowie verschiedene Servertypen (normale Server, PvP-Server, Rollenspielserver und Rollenspiel PvP-Server), Sprachchats etc. Spieler können alleine oder in Gruppen auf Missionen gehen, in Raids miteinander kämpfen, und in der Arena und auf Schlachtfeldern (Spieler von mehreren Servern in Gruppen) gegeneinander antreten .

Die in den Webforen diskutierten Kritikpunkte wie z. B. Langeweile sind für Hersteller alarmierend, da diese einen Abschwung des Lebenszyklus ihres Produktes signalisieren. Hier sind Hersteller gefordert, Ideen aufzugreifen und neue Inhalte zu schaffen. Verbindungsprobleme können zwar durchaus durch die Internetverbindung des Spielers bedingt sein und liegen oft außerhalb des Handlungsfelds der Hersteller, doch können Server auf dem neuesten Stand gehalten werden oder neue Server hinzugefügt werden: Um beispielsweise das Universum von EVE Online zu ermöglichen, in dem sämtliche Spieler auf einem Server spielen, betreibt der Hersteller CCP Games den größten Superrechner-Cluster in der Spieleindustrie.

Unter praxisorientierten Gesichtspunkten ermöglichen es Online Communities für Marketingmanager auf vorhandenes Material, welches in homogenen Nutzergruppen produziert wird, aufzugreifen und darauf - ähnlich wie bei
Fokusgruppendiskussionen oder traditionellen qualitativen Forschungsmethoden - für Marketingzwecke aufzubauen. Die Forumsdiskussionen sind hochaktuell und spiegeln damit bestmögliches qualitatives Entscheidungsmaterial für Produktentwicklungs- und variationsentscheidungen wieder. Sollten sich in der Online Community gewisse Experten oder Meinungsführer identifizieren lassen, so können Firmen und deren Produktmanager einfach mit diesen in Kontakt treten und für allfällige weitere Themen interiviewen.

Diese textanalytische Vorgangsweise stellt zweifellos nur eine von mehreren möglichen Variante der Erforschung von Online Communities dar. Beispielsweise könnten Firmen direkt im Spiel mitwirken, und dadurch unmittelbares, beobachtbares Erfahrungswissen generieren. Zwar ist es für derartige Arbeit unter forschungsethischen Gesichtspunkten wichtig diese Vorgangsweise mit den Spielern abzuklären, doch die Glaubwürdigkeit und Authentizität des Untersuchungsmaterials könnte damit noch gesteigert werden. Eine weitere Verbesserungsmöglichkeit für die gegenständliche Vorgangsweise stellt Triangulation dar. Dabei könnten in Zukunft durch den zusätzlichen Einsatz von Interviews und Fokusgruppen parallel, Qualitätsvorteile erzielt werden. Es wäre insbesondere möglich die Untersuchungsergebnisse von mehreren Seiten analytisch zu begründen und alternative Szenarien für Managemententscheidungen zu identifizieren.

Danksagung Die Autoren danken Stefan Kliment und Yen-Ting Lai für ihre Unterstützung in der Datensammlung und -analyse, sowie Noemi Pezderka und Matthias Hossinger für ihre konstruktive Kritik und Unterstützung zur Weiterentwicklung von früheren Entwürfen dieses Artikels.

\section{Literatur}

Aghamanoukjan A, Buber R, Meyer M (2007) Qualitative Interviews. In: Buber R, Holzmüller HH (Hrsg) Qualitative Marktforschung Theorie, Methode, Analyse. Gabler, Wiesbaden

Anderson WT, Challagalla GN, McFarland RG (1999) Anatomy of exchange. J Market Theory Pract 7(4):8-19

Andrews D, Preece J, Turoff M (2002) A conceptual framework for demographic groups resistant to on-line community interaction. Int J Electron Comm 6(3):9-24

Armstrong A, Hagel J (1996) The real value of on-line communities. Harvard Bus Rev 74(3):134-141

Bagozzi RP, Dholakia UM (2002) Intentional social action in virtual communities. J Interac Mark 16(2):2-21

Balasubramanian S, Mahajan V (2001) The economic leverage of the virtual community. Int J Electron Comm 5(3):103-138

Beckmann SC, Langer R (2007) Netnographie. In: Buber R, Holzmüller HH (Hrsg) Qualitative Marktforschung - Theorie, Methode, Analyse. Gabler, Wiesbaden

Blanchard AL (2008) Testing a model of sense of virtual community. Comput Hum Behav 24(5):2107-2123

Brown J, Broderick AJ, Lee N (2007) Word of mouth communication within online communities: conceptualizing the online social network. J Interac Mark 21(3):2-20 
Casaló LV, Flavián C, Guinalíu M (2008) Fundaments of trust management in the development of virtual communities. Manage Res News 31(5):324-338

Chiu CM, Hsu MH, Wang ETG (2006) Understanding knowledge sharing in virtual communities: an integration of social capital and social cognitive theories. Decis Support Syst 42(3):1872-1888

Cropanzano R, Mitchell MS (2005) Social exchange theory: an interdisciplinary review. J Manage 31(6):874-900

Dholakia UM, Bagozzi RP, Klein Pearo L (2004) A social influence model of consumer participation in network- and small-groupbased virtual communities. Int J Res Mark 21(3):241-263

Emerson RM (1976) Social exchange theory. Annu Rev Sociol 2:335-362

England D, Gray P (1998) Temporal aspects of interaction in shared virtual worlds. Interact Comput 11(1):87-105

Franke N, Piller FT (2003) Key research issues in user interaction with user toolkits in a mass customisation system. Int J Technol Manage 26(5/6):578-599

Franke N, Piller P (2004) Value creation by toolkits for user innovation and design: the case of the watch market. J Prod Innovat Manag 21(6):401-415

Füller J, Bartl M, Ernst H, Mühlbacher H (2006) Community based innovation: how to integrate members of virtual communities into new product development. Electro Commer Res 6(1):57-73

Füller J, Jawecki G, Mühlbacher H (2007) Innovation creation by online basketball communities. J Bus Res 60(1):60-71

Ghauri PN, Firth R (2009) The formalization of case study research in international business. der Markt (in Druck)

Ghauri PN, Grønhaug K (2005) Research methods in business studies: a practical guide, 3rd ed. Financial Times Prentice Hall, London

di Gregorio S (2007) Software-Instrumente Zur Unterstützung Qualitativer Analyse. In: Buber R, Holzmüller HH (Hrsg) Qualitative Marktforschung - Theorie, Methode, Analyse. Gabler, Wiesbaden

Gu B, Konana P, Rajagopalan B, Chen HWM (2007) Competition among virtual communities and user valuation: the case of investing-related communities. Inform Syst Res 18(1):68-85

Guba EG, Lincoln YS (1989) Fourth generation evaluation. Sage Publications, Newbury Park, CA

Hagel J, Armstrong A (1997) Net gain: expanding markets through virtual communities. Harvard Business School Press, Boston

Hemetsberger A (2002) Fostering cooperation on the internet: social exchange processes in innovative virtual consumer communities. Adv Consum Res 29(1):354-356

von Hippel E (2001) Perspective: user toolkits for innovation. J Prod Innovat Manag 18(4):247-257

von Hippel E, von Krogh G (2003) Open source software and the "private-collective" innovation model: issues for organization science. Organ Sci 14(2):209-223

Hitzler R (2007) Ethnographie. In: Buber R, Holzmüller HH (Hrsg) Qualitative Marktforschung - Theorie, Methode, Analyse. Gabler, Wiesbaden

Hoffman DL, Novak TP (1996) Marketing in hypermedia computermediated environments: conceptual foundations. J Marketing 60(3):50-68

Hsu CL, Lu HP (2007) Consumer behavior in online game communities: a motivational factor perspective. Comput Hum Behav 23(3):1642-1659

Jang H, Lorne O, Ko I, Koh J, Kim K (2008) The influence of on-line brand community characteristics on community commitment and brand loyalty. Int J Electron Comm 12(3):57-80

Koh J, Kim YG (2003) Sense of virtual community: a conceptual framework and empirical validation. Int $\mathrm{J}$ Electron Comm 8(2):75-93

Kollock P, Smith M (1999) Communities in cyberspace. Routledge, London
Kozinets RV (1999) E-tribalized marketing?: The strategic implications of virtual communities of consumption. Eur Manage $\mathrm{J}$ 17(3):252-264

Kozinets RV (2002) The field behind the screen: using netnography for marketing research in online communities. J Marketing Res 39(1):61-72

Kuckartz U (2007) Computergestützte Analyse Qualitativer Daten. In: Buber R, Holzmüller HH (Hrsg) Qualitative Marktforschung Theorie, Methode, Analyse. Gabler, Wiesbaden

Marschan-Piekkari R, Welch C (eds) (2004) Handbook of qualitative research methods for international business. Edward Elgar, Cheltenham, UK

Mennecke B, Terando WD, Janvrin DJ, Dilla WN (2007) It's just a game, or is it? Real money, real income, and real taxes in virtual worlds. Communications of AIS, 19. http://ssrn.com/abstract= 1022064. Letzter Zugriff 20. März 2009

Miller KD, Fabian F, Lin SJ (2009) Strategies for online communities. Strategic Manage J 30(3):305-322

Muniz AM Jr, O'Guinn TC (2001) Brand community. J Consum Res 27(4):412-432

Okoli C, Pawlowski SD (2004) The Delphi method as a research tool: an example, design considerations and applications. Inform Manage 42(1): 15-29

Penz E (2007) Paradoxical effects of the internet from a consumer perspective. Crit Perspecs Int Bus 3(4):364-380

Penz E, Sinkovics RR (2005) Formalisierung Qualitativer Internationaler Marketingforschung - Grundsätze und Anwendungsfall. In: Holzmüller HH, Schuh A (Hrsg) Innovationen Im Sektoralen Marketing - Festschrift Zum 60. Geburtstag von Fritz Scheuch. Physica Verlag, Heidelberg

Preece J (2000) Online communities: supporting sociability, designing usability. Wiley, New York

Rheingold H (1993) The virtual community: homesteading on the electronic frontier. Secker and Warburg, London

Richards L (2000) Using NVivo in qualitative research, 2nd ed. QSR International Pty., Bundoora, Victoria, Australia

Ridings CM, Gefen D, Arinze B (2002) Some antecedents and effects of trust in virtual communities. J Strategic Inf Syst 11(3-4):271-295

Robins K (1996) Cyberspace and the world we live in. In: Dovey J (ed) Fractal dreams: new media in social context. Lawrence \& Wishart, London

Rowe G, Wright G (1999) The Delphi technique as a forecasting tool: issues and analysis. Int J Forecasting 15(4):353-375

Schuler D (1996) New community networks: wired for change. Addison-Wesley, Reading, MA

Sinkovics RR, Penz E (2007) Mehrsprachige Interviews und Softwaregestützte Analyse - Problemlösungen und Implementierung mit Nvivo7. In: Buber R, Holzmüller HH (Hrsg) Qualitative Marktforschung - Theorie, Methode, Analyse. Gabler, Wiesbaden

Sinkovics RR, Penz E, Ghauri PN (2005) Analysing textual data in international marketing research. Qual Market Res Int J 8(1):9-38

Sinkovics RR, Penz E, Ghauri PN (2008) Enhancing the trustworthiness of qualitative research in international business. Manage Int Rev 48(6):689-714

Steinke I (2007) Die Güte Qualitativer Marktforschung. In: Buber R, Holzmüller HH (Hrsg) Qualitative Marktforschung - Theorie, Methode, Analyse. Gabler, Wiesbaden

Wasko McLure M, Faraj S (2005) Why Should I share? Examining social capital and knowledge contribution in electronic networks of practice. MIS Quart 29(1):35-57

Winkler K, Mandl H (2004) Virtuelle Communities - Kennzeichen, Gestaltungsprinzipien und Wissensmanagement-Prozesse. Ludwig-Maximilians-Universität, München 